\title{
Les entrées en matière non théoriques dans des mémoires de master et des articles de revues
}

\author{
Bertrand Daunay ${ }^{1}$ \\ Université de Lille, Laboratoire Théodile-CIREL, France \\ Daniel Bart ${ }^{2}$ \\ Université de Lille, Laboratoire Théodile-CIREL, France
}

Résumé : L'article veut contribuer à la description des spécificités du discours scientifique en comparant des entrées en matière de mémoires de master d'étudiants et d'articles d'enseignants-chercheurs. Dans ces entrées en matières, est interrogée la place des références à d'autres univers que le champ théorique, ce qui constitue l'une des questions sensibles dans l'apprentissage de l'écrit académique par de jeunes chercheurs. La comparaison porte sur les items identifiés comme réalisant une référence non théorique dans un corpus de 20 mémoires de master en didactique et de 20 articles issus de 7 revues de didactique. Cette étude exploratoire présente certaines convergences formelles des références non théoriques réalisées dans les écrits des chercheurs et des étudiants mais montre une différence quant au type de référence mobilisé. Cette analyse se double d'une intention pratique, les résultats de la comparaison donnant lieu à des activités destinées à aider les apprenants à mieux identifier les attentes académiques.

Mots-clés: Apprentissage du discours scientifique; Didactique; Entrée en matière; Littéracies universitaires; Références théoriques.

Título: As introduções não teóricas em dissertações de mestrado e artigos de pesquisa

Resumo: $O$ artigo se propõe a descrever as especificidades do discurso científico, comparando introduções de dissertações de mestrado e de artigos de professores doutores. Investiga-se com base nessas introduções o papel das referências feitas a outros campos do que ao teórico, o que constitui uma das dificuldades na aprendizagem da escrita acadêmica dos jovens pesquisadores. A comparação diz respeito a itens de referência não teórica em um corpus de 20 dissertações de mestrado em didática e de 20 artigos publicados em 7 revistas em didática. Esta análise exploratória traz à tona algumas convergências formais das referências não teóricas realizadas nos textos dos professores e dos estudantes, mas mostra uma diferença com relação ao tipo da referência utilizada. A análise é associada a um objetivo prático, pois os resultados da comparação dão lugar a atividades para ajudar os alunos a identificar melhor as expetativas acadêmicas.

\footnotetext{
${ }^{1}$ Professeur, université de Lille. Site professionnel : https://pro.univ-lille.fr/bertrand-daunay/. ORCID : https://orcid.org/0000-0002-3169-8515.

E-mail : bertrand.daunay@univ-lille.fr

${ }^{2}$ Maitre de conférences, université de Lille. Site professionnel : https://pro.univ-lille.fr/daniel-bart/.

E-mail : daniel.bart@univ-lille.fr
} 
Palavras-chave: Aprendizagem do discurso científico; Didática; Introdução; Letramentos acadêmicos; Referências teóricas.

Title: Non-theoretical introductions of Master dissertations and researchers' articles

Abstract : This paper would contribute to describe the specificities of the scientific speech, by the comparison of the introductions of Master dissertations and researchers' articles. In these introductions, we analyse more specifically the roles of the references to other universes than the theoretical field, which is one of the difficulties in the learning of the academic writing by young researchers. The comparison concerns some items realizing a nontheoretical reference in a corpus of 20 didactics Master dissertations and 20 articles stemming from 7 didactics journals. This exploratory study shows a convergence between the researchers' and the students' papers concerning the size and the shape of the nontheoretical references but highlights a difference as for the mobilized types of references. The description is coupled with a practical intention: the results of the comparison give rise to activities intended to help the learners to build themselves more precise knowledge about the academic expectations.

Keywords: Scientific Discourse Learning; Academic Literacies; Didactics; Introduction; Theoretical References.

\section{Introduction}

Ces dernières années ont vu se développer un important projet collectif de description des spécificités du discours scientifique, entendu (BOCH ; RINCK, 2010, p. 5) comme " discours produit dans le cadre de l'activité de recherche à des fins de construction et de diffusion du savoir " (pour une réflexion sur le choix des termes accompagnée d'un parcours des recherches en la matière, cf. GROSSMANN, 2017b). Dans cette entreprise, la comparaison est un outil empirique et théorique important, ce qui peut aisément s'expliquer : cerner un objet, si l'on veut éviter les essentialisations à priori (DETIENNE, 2000), nécessite de s'intéresser à ce qu'il peut bien ne pas être ou n'être pas exactement... Plusieurs comparaisons ont ainsi pu être effectuées entre discours proches, par exemple académique et professionnel (BOLÍVAR ; PARODI, 2015 ; MOIRAND, 1995 ; RUSSELL, 2012) ; académique et juridique (KANTÉ, 2010) ; scientifique et didactique (DAUNAY ; DELCAMBRE, 2017). La comparaison sert également à mieux caractériser certaines dimensions du discours scientifique lui-même : pour simplement prendre quelques exemples qui nous paraissent illustratifs, évoquons les comparaisons entre pays (BAILLY ; ALVES ASSIS ; DENEI, 2016 ; CORACINI, 1992 ; DONAHUE, 2008 ; FL ØTTUM ; DAHL ; KINN, 2006) ; entre langues (FLØTTUM ; DAHL ; KINN, 2006 ; VOLD, 2008) ; entre oral et écrit (JACQUES, 2017); entre disciplines (DELCAMBRE; LAHANIER-REUTER, 2010 ; FLØTTUM ; DAHL ; KINN, 2006 ; HYLAND ; BONDI ; REUTNER ; SCHWARZE, 2008 ; RINCK, 2006 ; VOLD, 2008) ; entre niveaux d'étude (DONAHUE, 2010 ; DELCAMBRE ; LAHANIER-REUTER, 2010), entres experts et débutants (BOCH ; GROSSMANN, 2002 ; FLØTTUM ; VOLD, 2010 ; RINCK ; POUVREAU, 2009)... 
C'est à cette dernière catégorie que nous voulons apporter une contribution, par la comparaison de textes d'étudiants et d'enseignants-chercheurs, en doublant notre approche descriptive d'une intention pratique ; en effet, nous plaçant dans une perspective didactique, nous souhaitons faire de la comparaison un moyen de construction de connaissances dans les deux sens du terme: au sens scientifique de l'expression, nous espérons apporter, par la comparaison, des éléments nouveaux qui participent à la construction collective cumulative des connaissances sur l'objet ; au sens d'une pratique didactique, nous pensons qu'une telle comparaison peut aider les apprenants à se construire pour eux-mêmes des connaissances plus précises sur les attentes des institutions qui les accueillent en matière de normes académiques. Cette double approche, qui illustre à sa manière le lien étroit entre recherche et enseignement, qui est censé présider aux études universitaires, fera des étudiants à la fois des objets de l'étude (quand leurs pratiques scripturales seront comparées à celle d'autres objets, celles des chercheurs expérimentés) et des sujets de l'analyse (en tant qu'elle vise leur appropriation d'un savoir nouveau).

La comparaison que nous présentons ici porte sur les entrées en matière des écrits académiques de mémoires de master et d'articles publiés dans des revues reconnues. Par entrée en matière, nous entendons la première subdivision du texte (délimitée explicitement comme telle par son auteur), par laquelle le scripteur entre - et fait entrer le lecteur - dans son écrit. Cette désignation, plutôt que celle d'introduction, nous a paru plus adaptée pour tenir compte des organisations textuelles variables de notre corpus: certains écrits ne comportaient ainsi pas d'introduction mais commençaient par une première partie tandis que dans d'autres, la première subdivision explicite pouvait segmenter une sous-partie de l'introduction.

Dans ces entrées en matière, nous interrogeons plus précisément la place du discours non théorique, par la référence à d'autres univers que le champ théorique. Ce choix tient à notre intérêt pour le fait que cette question constitue une difficulté particulièrement marquée de l'apprentissage de l'écrit académique par de jeunes chercheurs.

Après avoir présenté les principes qui sous-tendent notre projet (deuxième partie), nous présenterons la méthodologie de notre travail de comparaison (troisième partie) puis ses résultats (quatrième partie), pour terminer (cinquième partie) par des propositions d'activités fondées sur le corpus et les résultats de notre analyse. 


\section{L'objet de la comparaison : l'entrée en matière}

\section{Positionnements}

"Les écrits scientifiques sont souvent considérés comme un genre dépersonnalisé, avec un fort effacement énonciatif, où l'auteur se dissimule derrière la présentation de faits objectifs et des modalités de raisonnement partagées par la communauté scientifique ", notent Tutin et Grossmann (2013, p. 13), pour se démarquer d'une telle position. À l'appui de travaux divers (SWALES, 1990 ; FLØTTUM ; DAHL ; KINN, 2006 ; RINCK, 2006), ils rappellent que « ce constat est à nuancer, en tout cas dans certaines disciplines ", du fait de la dimension rhétorique d'un texte dont l'intention argumentative est importante (cf. POLLET ; GLORIEUX, 2016).

De fait, par-delà les normes du discours de recherche qui proscrivent une certaine expression du scripteur - comme sujet biographique, social, politique, doté de motivations, d'opinions, d'affectivité - au profit d'une neutralité du sujet théorique ou épistémique, les travaux cités, parmi de nombreux autres (pour d'autres références, voir BOCH ; RINCK, 2010, p. 6 sq.) ont pu montrer que l'écriture scientifique comporte des manifestations spécifiques de subjectivité.

Cela peut sembler plus particulièrement vrai dans l'entrée en matière, « lieu propice à la circulation des discours ", comme le disent Boch, Grossmann et Rinck (2010) du cadrage théorique, dans la mesure où l'on peut voir là, comme Tutin (2010) le dit des introductions et des conclusions, des « parties textuelles peu techniques où le positionnement de l'auteur est particulièrement marqué "(p. 16 sq). Le terme de positionnement qu'utilise Tutin est intéressant pour tenter d' " étayer le type de subjectivité auquel on a affaire " (BOCH ; RINCK, 2010, p. 7), car il permet d'englober diverses modalités d'expression du sujet scripteur dans son texte.

Outre les marques linguistiques $\mathrm{du}$ positionnement (pronoms personnels ou modalisateurs) qui ont donné lieu à de nombreux travaux dans le champ de l'étude du discours scientifique et que Grossmann (2017a) évoque, le positionnement nous semble pouvoir s'identifier par ce à quoi se réfère le scripteur dans son texte. En effet, loin de se référer aux seuls auteurs théoriques, notamment dans son entrée en matière, le scripteur peut aussi faire référence à son expérience professionnelle ou personnelle, à des discours médiatiques ou prescriptifs, aux enjeux institutionnels de sa recherche, et à bien d'autres choses encore.

Si ces références non théoriques ne sont pas privilégiées dans les normes de l'écriture académique, quand elles ne sont pas proscrites, une pratique ordinaire de lecture de textes scientifiques dans diverses disciplines des sciences humaines fait facilement apparaitre qu'on 
en trouve de manière régulière. Elles peuvent prendre place à n'importe quel endroit de l'entrée en matière et peuvent donc être une des modalités rhétoriques des divers passages obligés de l'introduction tels qu'ils sont décrits par Swales (1990, p. 141-142), même si l'on peut considérer que c'est le mouvement de constitution d'un territoire ("Establishing a Territory "), et plus particulièrement la phase de revendication de l'importance du sujet de I'article ("Claiming centrality »), qui est le plus propice aux énoncés non théoriques (cf., dans une perspective rhétorique : HERMAN, 2017).

Toutefois, il nous semblait, intuitivement, que ces références-là étaient malgré tout plutôt cantonnées, en tout cas non systématiques dans les textes d'auteurs expérimentés, alors que c'était une pratique spontanée fréquente dans les premiers écrits d'étudiants de master que nous formions à l'écriture de recherche ${ }^{3}$. C'est cette intuition que nous voulions étayer par une étude comparatiste de corpus contrastés, afin d'identifier la place qu'y prennent les références non théoriques et les modalités rhétoriques qui permettent de rendre ces références plus ou moins acceptables.

\section{Une approche comparatiste}

Nous avons choisi de travailler, dans le champ théorique spécifique de la didactique, sur deux écrits qui nous semblent emblématiques : le mémoire de master, qui sanctionne un cycle d'initiation à la recherche, et l'article de recherche, modèle-type du discours scientifique validé. Cette présentation dichotomique est évidemment un peu rigide et néglige les variations internes à ces deux catégories comme leurs évolutions historiques : les normes de chacune de ces catégories de textes peut varier d'une discipline à l'autre, y compris au sein des didactiques, voire d'un enseignant-chercheur à l'autre, et on ne saurait négliger que la notion d'article ou de mémoire de recherche peut recouvrir des réalités diverses - sur le plan de la forme, des normes discursives attendues, des modalités d'évaluation des textes, etc. De telles variations interrogent, comme le montre Donahue (2010) la notion d'expertise en matière de discours théorique.

Questionnant le statut et la forme du discours théorique d'autrui dans «l'écrit de recherche en formation », Reuter (2001, p. 14) précise que

\footnotetext{
${ }^{3}$ Ce que nous nous expliquions par la nécessaire acculturation progressive des étudiants à une forme de rhétorique académique qui trouve, dans le jeu des références bibliographiques et du positionnement du scripteur, un point d'achoppement particulièrement visible dans l'introduction, comme le montre l'étude d'un cas par Pasquotte-Vieira et Salek Fiad (2015).
} 
ce qui est analysé comme un discours autre dans le discours théorique du "jeune » chercheur n'est en réalité qu'une des formes discursives "familières " échappant au contrôle du discours de recherche, vécu en début de carrière (voire pendant longtemps) comme de l'altérité.

Présentant des exemples d'articulations problématiques entre des discours théoriques et non théoriques, l'auteur (REUTER, 2001, p. 15) interroge notamment « les formes recevables de mélanges des discours, sachant qu'excessivement rares sont les discours purement formels et homogènes (et que ces traits ne sont d'ailleurs pas des cautions en matière de recherche) ".

C'est avec à l'esprit la nécessaire prudence qu'exige l'approche comparatiste, si l'on ne veut éviter qu'elle réifie à son tour ce qu'elle cherche à dénaturaliser, que nous rendrons compte de notre étude exploratoire, sachant qu'elle interroge les normes actuelles du discours de recherche dans un champ restreint, celui de la didactique. Notre comparaison d'entrées en matière de mémoires de master et d'articles dans des revues reconnues du champ est destinée à identifier les différences entre apprentis chercheurs et chercheurs confirmés, dans la mesure où l'on peut supposer que le temps a quelque influence sur la rhétorique de l'écrit de recherche. Cette influence n'est sans doute pas linéaire : si l'acculturation à des normes peut tendre à une rigidification de celles-ci (la place donnée aux énoncés non théoriques tendrait progressivement à diminuer), leur maitrise peut au contraire laisser une certaine latitude (qui pourrait laisser des positionnements non théoriques reprendre une place chez le scripteur aguerri) et donner lieu à une plus grande acceptabilité.

De fait, disons-le tout de suite : nous verrons que nos premières explorations montrent que l'usage de références à des champs d'expérience non théoriques dans les entrées en matière analysées n'est pas anecdotique, du point de vue quantitatif et que l'hypothèse, à l'origine de ce travail, d'un usage de ces références plus fréquent dans les mémoires de master que dans les articles ne se vérifie pas nettement : les résultats tendent en effet à montrer une certaine convergence entre les écrits des chercheurs et des étudiants. Nous verrons néanmoins que des analyses plus fines permettent de faire apparaitre des différences, qui tiennent surtout au type de référence mobilisé.

\section{Une approche didactique}

Nous l'avons dit, notre approche est didactique. Mais il convient d'emblée de lever une possible ambigüité : nous avons employé jusqu'ici le mot didactique pour désigner un champ théorique. Et c'est dans cette acception qu'il faut continuer à l'entendre ! Mais la didactique étant une discipline de recherche qui analyse les contenus d'enseignement et 
d'apprentissage, c'est bien ici un contenu d'enseignement et d'apprentissage que nous visons : l'entrée en matière dans un mémoire de master en didactique.

Et c'est à la fois la construction théorique et méthodologique de notre étude et son exploitation possible en cours qui sont didactiques, dans la logique d'une didactique des disciplines universitaires telle qu'elle doit encore s'inventer (DELCAMBRE, 2016). Née d'un questionnement professionnel, cette étude trouvera son débouché dans une exploitation professionnelle. La comparaison, dès lors, a deux vertus, toutes deux didactiques - I'une théorique et l'autre pratique : elle permet en effet d'un côté de (dé)construire l'objet entrée en matière de manière contrastive en identifiant les variations et les invariants d'un genre selon deux contextes de réalisation (c'est là la logique d'une didactique comparée) ; d'un autre côté, la comparaison devient, selon une démarche très classique, un outil d'enseignement et d'apprentissage, pour faire découvrir ce qui peut caractériser un genre que les étudiants ne découvrent pas vraiment, mais dont ils approfondissent la connaissance, notamment en conscientisant ce qui était implicite et, le cas échéant, en remaniant des représentations qui pouvaient les éloigner des attentes académiques.

La présentation que nous allons faire de notre étude est donc à lire pour elle-même, mais aussi comme possible objet de discussion avec les étudiants, de plusieurs points de vue : nous présenterons en effet, dans la quatrième partie, des activités qui solliciteront à la fois le corpus, les résultats et la méthodologie.

\section{Méthodologie d'une comparaison entre entrées en matière d'articles et de mémoires}

Faute de pouvoir présenter dans le détail la méthodologie de notre étude, nous donnerons ci-dessous toutes les informations que nous croyons nécessaires à la compréhension de notre démarche.

\section{La constitution du corpus}

Rappelons que notre corpus est constitué d'entrées en matière de mémoires de master et d'articles dans des revues reconnues du champ didactique. Délimiter une entrée en matière n'est pas simple, du fait de la diversité des formats possibles; aussi avons-nous fait un choix arbitraire mais rationnel : nous considérons comme entrée en matière la première subdivision du texte proposée par le scripteur, c'est-à-dire le début du texte jusqu'à son premier titre. Cette manière de faire dévolue finalement aux scripteurs la délimitation de notre objet, ce qui 
a l'inconvénient de montrer une disparité (relative) des items de notre corpus mais a l'avantage de se fonder sur le geste d'écriture propre au scripteur dans sa démarche d'entrée en matière.

Pour limiter notre corpus dans un premier temps, en vue de l'étude exploratoire que nous présentons ici, nous avons tiré au sort 20 mémoires de master en didactique de l'université de Lille $^{4}$ et 20 articles issus de 7 revues de didactique ${ }^{5}$; tous les textes du corpus ont été réalisés ou publiés entre 2010 et 2016.

Dans la suite de l'article, des codes du type A1-3 ou M5-2 identifient les éléments du corpus : les lettres $\mathrm{A}$ ou $\mathrm{M}$ signifient article ou mémoire, le premier chiffre indique le numéro du texte concerné et le second, le numéro de la référence non théorique relevée par nos soins.

Le corpus, pour limité qu'il soit, reste important et totalise une centaine de pages. Le corpus mémoires (de 156498 signes tout compris) est quasiment deux fois plus long que le corpus articles (85 527 signes) et il faut compter une grande disparité de longueur entre les écrits (de quelques pages à quelques dizaines de lignes, voire moins pour un article).

Notre corpus, par définition, ne saurait être anonyme, puisqu'il s'agit de textes publics - et publiés pour ce qui est des articles. Pour autant, nous ne donnons pas la liste de nos sources, à la fois par manque de place et pour éviter toute mésinterprétation d'une désignation ${ }^{6}$.

Partant de ces 40 entrées en matière de mémoires et d'articles, et, nous avons délimité les items que nous avons identifiés comme réalisant une référence non théorique. De façon classique, nous avons considéré comme item un passage encadré par deux ponctuations fortes (i.e. les signes de ponctuation suivis d'une majuscule mais aussi le point-virgule et, plus rarement, en fonction du contexte, le double point) ${ }^{7}$. Dans notre démarche, une note de bas de page qui informe un énoncé catégorisé comme item est considérée comme appartenant à

\footnotetext{
${ }^{4}$ Parmi ceux qui, ayant obtenu au moins la note de $14 / 20$, sont archivables à la bibliothèque du département des sciences de l'éducation (il s'agit donc de documents publics).

${ }^{5}$ Les revues sont les suivantes : Éducation et didactique ; Recherches en didactique des sciences et des technologies; Repères. Recherches en didactique du français langue maternelle; Recherches en didactique des mathématiques; Recherches en didactiques; Pratiques. Linguistique, littérature, didactique ; Recherches. Revue de didactique et de pédagogie du français. Sont exclus du corpus les recensions, les entretiens, les textes de présentation (de thèmes, rubriques, etc.) qui n'ont pas le format d'article, les « réponses » d'auteur, etc.

${ }^{6}$ Nous sommes redevables aux auteur.e.s des textes que nous avons exploités de nous avoir permis de mener ce travail. Sans pouvoir les nommer, nous leur exprimons ici toute notre reconnaissance. Nous remercions également Marie-Michèle Cauterman, Isabelle Delcambre et Christiane Donahue pour leurs conseils avisés sur des versions antérieures de ce texte.

${ }^{7}$ La délimitation des items a été faite par chacun de nous séparément et stabilisée par un accord commun. En raison de la dimension exploratoire de notre étude et des rares cas de divergence dans cette tâche de délimitation, nous n'avons pas eu recours pour ce travail de codage à des tests statistiques de concordance entre codeurs.
} 
cet item : dans ce cas, le texte en note est pris en compte dans les dénombrements de signes de l'item. En revanche, certaines notes peuvent être considérées comme des énoncés autonomes.

Les références non théoriques

Par références non théoriques nous entendons des références à des champs d'expérience autres que les discours d'auteurs théoriques. Nous n'entrerons pas dans des considérations sur les implications ontologiques des conceptions théoriques de la référence (cf. KLEIBER, 1997): sans vouloir produire une théorie de la référence, nous nous plaçons dans une perspective empirique : en gros, à quoi d'extérieur à lui-même renvoie le discours pour entrer en matière ? L'objectif pour nous était d'identifier, dans les textes de notre corpus, les renvois à un extérieur au discours qui ne soit pas une référence à un auteur théorique.

La construction de notre grille d'analyse s'est appuyée sur de multiples lectures de notre corpus, individuelles et croisées, accompagnées de tests de différentes catégorisations possibles. Au final, nous avons retenu 12 catégories de référence, présentées ci-dessous sans les commenter quand elles nous semblaient transparentes et, surtout, sans pouvoir les exemplifier. Cela aurait permis de préciser nos choix et, sans doute, de clarifier notre propos, mais la place que cela demandait excédait largement les limites de cet article.

- Référence aux pratiques d'un champ professionnel ou plus généralement à des pratiques sociales.

- Référence à un discours médiatique.

- Référence « historique » non référencée à un historien.

- Référence à un intérêt personnel (privé, professionnel ou académique).

- Référence au contexte académique (formation ou recherche).

Ces deux dernières catégories (référence à un intérêt personnel et au contexte académique de l'écrit produit) peuvent parfois être liées, mais nous avons cherché à les distinguer au mieux (pour une autre catégorisation de la manière dont le sujet auteur apparait dans l'explicitation de l'histoire de son mémoire ou de sa recherche, voir CRINON ; GUIGUE, 2002).

- Référence à un champ de recherche large, sans référence d'auteur.

Cette catégorie peut surprendre, car il s'agit bien d'une référence théorique, mais nous l'avons intégrée car elle fonctionne de manière assez semblable aux autres. II s'agit d'une manière large d' " ancrer son objet dans le paysage théorique " (BOCH ; GROSSMANN ; RINCK, 2006), mais sans référence à un auteur: on est dans le cas d'une "référence générique » (CORACINI, 1992). 
- Référence à un contexte institutionnel.

L'exemple typique dans le domaine didactique est sans doute les instructions officielles, quand elles ne sont pas convoquées à titre de corpus d'étude (à ce sujet, voir les précisions données ci-dessous).

- Référence à des valeurs, jugement de valeur (autres que ceux portés sur des objets théoriques).

- Référence à un discours commun (non directement référé à un champ de pratiques).

Cette catégorie comprend toutes les références à ce qui peut sembler une "réalité » non interrogée, c'est-à-dire tout ce qui relève du sens commun et qui n'entre pas dans une des catégories précédentes.

- Référence littéraire (quand elle n'est pas un matériau empirique).

Nous comptons comme telle une référence à un récit fictionnel qui n'est pas traité comme corpus ni comme discours théorique.

- Référence au futur : prescription.

On pourrait considérer cela comme une entrée en matière prospective. Cette catégorie concerne tous les énoncés qui renvoient à la possibilité d'agir sur un champ social.

- Référence au lecteur.

Nous appelons ici "référence au lecteur " toute mention explicite du lecteur ou toute adresse directe à lui. Nous n'incluons pas ici les formes personnelles comme le « nous » ou le " on » (voir FL ØTTUM ; VOLD, 2010) ou des formes verbales correspondantes (comme, par exemple, "rappelons que »). Outre que ces marques sont très différentes de toutes les autres marques de références des autres catégories et que les inclure aurait déséquilibré cette catégorie par rapport aux autres, se posait l'épineuse " question de savoir qui est "nous" » (FRANÇOIS, 2012, p. 83)...

\section{Quelques précisions}

Dans les cas où les énoncés entrecroisent différentes catégories, nous avons retenu celle qui nous paraissait la plus nettement mise en avant.

Nos items comportent parfois des références théoriques : c'est que nous avons considéré qu'elles portaient sur un segment court et qu'elles n'engageaient pas l'ensemble de la proposition. Inversement, quand des propositions sans théorisation étaient précédées ou 
suivies de références théoriques qui les étayaient, nous ne les avons pas relevées comme items.

Nous excluons toutes les références qui concernent la recherche réalisée ou sa présentation annoncée: dans ces cas-là il y a bien une référence extérieure à l'extrait proprement dit, mais elle appartient au discours où s'insère ce dernier. Cela concerne toutes les "questions de recherche" ou les "hypothèses", de même que les précisions méthodologiques et les présentations du plan de l'écrit.

Nous excluons encore ce qui, dans les références à un extérieur au texte, relève du matériau empirique de l'étude. C'est ainsi que, dans un article (A18) portant sur l'écriture d'une œuvre littéraire, nous ne considérons pas les références à cette œuvre comme une référence extérieure, qu'on peut considérer comme « texte englobé » (CORACINI, 1992).

\section{Qu'en est-il des références non théoriques dans les entrées en matière d'articles et de mémoires?}

Nous avons déjà dit, dès l'introduction, que l'on ne pouvait pas voir de fortes différences entre les articles et les mémoires quant à l'importance quantitative des références non théoriques. II convient dans un premier temps d'affiner ce résultat pour montrer ensuite où se jouent davantage les différences : dans les modalités discursives de ces références ${ }^{8}$.

\section{Premières approches des références non théoriques dans les entrées en matière}

Le poids des références autres que théoriques n'est pas négligeable dans les entrées en matière du corpus. En rapportant le nombre de caractères de ces références au nombre total de caractères des entrées en matière, on constate en effet qu'elles représentent $38,5 \%$ des écrits étudiés.

Ce résultat se double d'un autre constat : il y a, à première vue, peu d'écart entre le poids du nombre de signes non théoriques calculé pour les deux types d'écrit; si l'on exclut de l'échantillon des entrées en matière qui ne contiennent aucune référence non théorique ( $A 5$,

\footnotetext{
${ }^{8}$ Les analyses que nous présentons dans la suite s'appuient des statistiques descriptives telles que des calculs de fréquences en pourcentages. II s'agit pour nous d'identifier des tendances manifestes de notre corpus. Compte tenu de la dimension exploratoire de notre étude et des caractéristiques de notre corpus, le recours à des tests de significativité, qu'aurait permis un corpus d'une autre ampleur, ne nous a pas semblé fondé.
} 
A18 et M14), les taux de références non théoriques sont proches : $41 \%$ pour les mémoires et $43 \%$ pour les articles, ce qui fait apparaitre une assez grande convergence des néophytes et des experts.

Une autre tendance partagée s'observe au niveau de la longueur des références analysées, si l'on calcule les médianes (sans compter les valeurs nulles) : on observe un écart d'une demiligne environ entre la médiane des références non théoriques des mémoires ( 280 signes) et celle des articles (333 signes). Toutefois, la proximité de ces valeurs médianes ne doit pas masquer une autre convergence formelle concernant les fortes variations de longueur des items relevés pour chacun des corpus. L'écart interquartile est ainsi de 456 signes pour les articles et de 442 signes pour les mémoires.

Enfin, cette convergence se manifeste également dans le nombre des énoncés non théoriques rapportés à la taille respective des corpus (sachant que le nombre de signes du corpus articles est environ deux fois moins important que celui du corpus mémoire) : 62 items relevés pour les articles, 136 pour les mémoires. On retrouve cette même stabilité du côté du nombre maximum d'items relevés pour chaque type d'écrit : 8 items maximum pour A7 (7128 signes) contre 16 pour M18 (15657 signes).

Ces premiers indicateurs tendent donc à souligner à la fois l'importance quantitative des références non théoriques dans les entrées en matière étudiées et des convergences formelles entre les mémoires et les articles. Ces explorations semblent indiquer que ces réalisations discursives dans les entrées en matière n'ont rien d'exceptionnel ni rien d'une écriture désordonnée ou maladroite. Il faut néanmoins chercher à voir d'une part en quoi nos catégories de référence contribuent diversement à ces modalités de discours et d'autre part $s^{\prime} i l y$ a des différences qui se manifestent dans le maniement de ces dernières pour nos deux corpus. C'est ce que nous allons nous attacher à faire dans la suite de cet article.

La distribution des catégories de références non théoriques : quelques spécificités selon les corpus

Notre grille d'analyse des références non théoriques comporte 12 catégories différentes, comme on l'a vu plus haut. Excepté les écrits dans lesquels nous n'avons pas identifié de référence, la médiane du nombre de ces catégories représentées par texte du corpus est de 3. Mais ce chiffre est deux fois plus élevé pour les mémoires (4) que pour les articles (2). À l'inverse, le nombre maximum de catégories différentes relevées ne varie pas beaucoup dans nos deux corpus : il est de 8 pour un mémoire (M18) et de 6 pour un article (A19, il s'agit d'une 
revue interface; ou 5 pour A2 et A4 publiés dans des revues de recherche). Toutes les catégories ne sont cependant pas également représentées parmi les items.

Au niveau global, le tableau 1 ci-dessous montre que, parmi ces 12 catégories, 6 peuvent être considérées comme très rares dans la mesure où chacune d'elles rassemble moins de $2,5 \%$ du nombre de signes des références non théoriques: les références à du discours commun $(2,1 \%)$, à un intérêt personnel $(1,8 \%)$, médiatiques $(1,5 \%)$, " historiques » $(1,3 \%)$, littéraires $(0,6 \%)$, à un lecteur $(0,5 \%)$.

Tableau 1. Le poids des catégories de références (en pourcentage du nombre de signes analysés)

\begin{tabular}{lcccccc}
\hline & \multicolumn{2}{c}{ Global } & \multicolumn{2}{c}{ Mémoires } & \multicolumn{2}{c}{ Articles } \\
\hline Références & $\begin{array}{c}\text { Nb de signes } \\
\text { (nb d'items) }\end{array}$ & $\begin{array}{c}\text { \% du nb } \\
\text { de signes }\end{array}$ & $\begin{array}{c}\text { No de } \\
\text { signes (nb } \\
\text { d'items) }\end{array}$ & $\begin{array}{c}\text { \% du nb } \\
\text { de } \\
\text { signes }\end{array}$ & $\begin{array}{c}\text { Nb de } \\
\text { signes (nb } \\
\text { d'items) }\end{array}$ & $\begin{array}{c}\text { \% du nb } \\
\text { de } \\
\text { signes }\end{array}$ \\
\hline Contexte académique & $30158(52)$ & 32,4 & $23142(42)$ & 36,6 & $7016(10)$ & 23,5 \\
Pratiques & $22680(43)$ & 24,3 & $19103(34)$ & 30,2 & $3577(9)$ & 12 \\
\hline Contexte institutionnel & $12206(28)$ & 13,1 & $7739(21)$ & 12,2 & $4467(7)$ & 14,9 \\
Champ de recherche large & $10256(25)$ & 11,0 & $2329(9)$ & 3,7 & $7927(16)$ & 26,5 \\
\hline Futur & $8128(14)$ & 8,7 & $4642(7)$ & 7,3 & $3486(7)$ & 11,7 \\
Valeurs & $2394(11)$ & 2,6 & $435(3)$ & 0,7 & $1959(8)$ & 6,6 \\
\hline Discours commun & $1938(7)$ & 2,1 & $1434(5)$ & 2,3 & $504(2)$ & 1,7 \\
Intérêt personnel & $1716(8)$ & 1,8 & $1587(7)$ & 2,5 & $129(1)$ & 0,4 \\
\hline Historique & $1424(2)$ & 1,5 & $406(3)$ & 0,6 & $842(2)$ & 2,8 \\
\hline Médiatique & $1248(5)$ & 1,3 & $1424(2)$ & 2,2 & & 100 \\
\hline Littéraire & $585(1)$ & 0,6 & $585(1)$ & 0,9 & & $29907(62)$ \\
\hline Lecteur & $485(2)$ & 0,5 & $485(2)$ & 0,8 & 100 & \\
\hline Total général & $93218(198)$ & 100 & $63311(136)$ & &
\end{tabular}

On peut se demander si ces 6 références les moins fréquentes ne constituent pas la limite de l'acceptabilité des références non théoriques dans l'écriture de recherche. Cela est plus vrai encore pour les références à un lecteur, littéraires et médiatiques qui ne sont pas représentées dans le corpus d'entrées en matière d'articles.

À l'inverse, sur l'ensemble des corpus, les deux premières catégories de référence rassemblent plus de la moitié $(56,7 \%)$ du nombre de signes des références non théoriques : les références à un contexte académique (formation ou recherche) $(32,4 \%)$ et les références aux pratiques d'un champ professionnel ou plus généralement social $(24,3 \%)$.

Il faut toutefois signaler que cette prédominance des catégories que nous venons d'observer d'un point de vue global ne se retrouve pas également dans les deux corpus : les 
références à un contexte académique $(36,6 \%)$ sont bien premières pour les mémoires tandis que ce sont les références à un champ de recherche large (26,5\%) pour les articles (cette catégorie ne rassemblant que $3,7 \%$ des signes analysés du côté des mémoires). Cette différence de catégorie de références majeure dans les deux corpus va dans un sens relativement attendu, sur lequel nous reviendrons dans la partie suivante. Dans cette perspective on pourrait également citer la différence de poids des références à des pratiques (30,2\% pour les mémoires ; $12 \%$ pour les articles). II faudrait bien sûr des recherches plus approfondies pour savoir si ces différences un peu caricaturales trouvent des confirmations dans l'analyse de corpus différents.

Du reste, si l'on aborde la question des catégories dominantes dans le tableau 1 sous l'angle du cumul des fréquences qui permettent d'atteindre ou de dépasser les trois quarts des signes pour les deux corpus, on observe que les distributions sont assez proches : pour les mémoires, il s'agit de l'association des références à un contexte académique $(36,6 \%)$, à des pratiques $(30,2 \%)$, à un contexte institutionnel $(12,2 \%)$; pour les articles, il s'agit du cumul des références à un champ de recherche large $(26,5 \%)$, à un contexte académique $(23,5 \%)$, à un contexte institutionnel $(14,9 \%)$ et à des pratiques (12\%).

Le poids de quatre catégories dans les entrées en matière étudiées est ainsi mis en évidence. Nous allons donc consacrer la partie suivante à une exploration plus fine de leur fonctionnement selon les corpus.

Quelques caractéristiques des principales catégories de références non théoriques selon les corpus

L'observation de la distribution des références dans le tableau 1 montre que les références à un contexte académique, à des pratiques et à un contexte institutionnel caractérisent les entrées en matière des mémoires par leur fréquence élevée en termes de nombre de signes.

Toutefois, se pencher, non plus sur le nombre de signes de ces références dominantes, mais sur leur présence ou leur absence, permet d'affiner ces résultats comme le montre le tableau 2 ci-dessous. 
Tableau 2. Nombre d'entrées en matière qui comportent au moins une des principales catégories de références

\begin{tabular}{lccc}
\hline Références & $\begin{array}{c}\text { Mémoires } \\
(\mathbf{n}=\mathbf{1 9 *})\end{array}$ & $\begin{array}{c}\text { Articles } \\
(\mathbf{n = 1 8 *})\end{array}$ & $\begin{array}{c}\text { Total } \\
(\mathbf{n}=\mathbf{3 7} *)\end{array}$ \\
\hline Pratiques & 17 & 7 & 24 \\
\hline Contexte académique & 14 & 7 & 21 \\
\hline Contexte institutionnel & 13 & 6 & 19 \\
\hline Champ de recherche large & 5 & 12 & 17 \\
\hline Futur & 7 & 5 & 12 \\
Valeurs & 3 & 6 & 9 \\
\hline Intérêt personnel & 6 & 1 & 7 \\
Discours commun & 5 & 2 & 7 \\
\hline Historique & 2 & 2 & 4 \\
Médiatique & 2 & & 2 \\
\hline Lecteur & 2 & & 2 \\
\hline Littéraire & 1 & & 1 \\
\hline
\end{tabular}

*Rappel : deux articles et un mémoire ne comportent pas de référence à du discours non théorique

Ce tableau confirme bien que la majorité des entrées en matière de mémoires présente au moins une référence à des pratiques d'un champ professionnel ou plus généralement social et ou à un contexte académique et ou à un contexte institutionnel. Mais il permet de faire apparaitre plus clairement le poids déterminant des références à des pratiques : 17 entrées en matière de mémoires (sur 19 qui contiennent au moins un item) font au moins une référence à des pratiques alors qu'on en trouve seulement sept du côté des articles. De plus, des traitements supplémentaires montrent que plus de la moitié $(n=9)$ de ces 17 entrées en matière de mémoires qui font au moins une référence à des pratiques font également référence à un contexte académique et à un contexte institutionnel.

On aurait peut-être, avec ces trois catégories, des marqueurs spécifiques d'entrée en matière relevant de "l'écriture de recherche en formation " (REUTER, 2004), dont l'auteur a montré qu'elle donnait lieu, notamment pour les scripteurs, à une tension particulière entre les postures d'apprenti-chercheur et de chercheur (cf. aussi DELCAMBRE ; LABORDE-MILAA, 2002 dans le cadre de mémoires professionnels).

C'est pour mieux décrire ce phénomène que nous avons distingué certains items sélectionnés avec un indice d'autoréférence permettant de caractériser les références qui mettent en avant l'expérience propre du scripteur. Or les résultats montrent que ces indices marquent essentiellement des items issus d'entrées en matière de mémoires. Sur les 61 indices de ce type que nous avons attribués, 49 l'ont été pour des références de mémoires. Et le fait que parmi ces 49 items, 39 soient des références à un contexte académique montre le poids du récit de recherche dans les entrées en matière des étudiants. 
Du côté des articles, nous l'avons vu avec le tableau 1, les entrées en matière sont, dans notre corpus, caractérisées par deux catégories dominantes (références à des champs de recherche large sans auteurs précis et à un contexte académique) et, dans une moindre mesure, par des références à un contexte institutionnel et à des pratiques. La contextualisation académique ou institutionnelle pourrait toutefois être considérée comme particulièrement importante dans ce corpus d'articles: un regroupement de ces deux catégories ressemblerait $38,4 \%$ du total des signes affectés à des références non théoriques.

Pourtant le tableau 2 permet de voir qu'en fait, les références un contexte institutionnel $(n=7)$ et ou académique $(n=7)$ ne concernent, comme les références à des pratiques $(n=7)$, qu'une minorité d'entrées en matière d'articles. Ces données permettent par conséquent de modérer l'importance de la contextualisation institutionnelle ou académique dans les entrées en matière d'articles que nous avions entrevue à l'aide du tableau 1 . Si ces références rassemblent un nombre de signes conséquent, elles n'apparaissent que dans un nombre réduit d'articles.

À l'inverse, le tableau 2 confirme que les références à un champ de recherche large dans les entrées en matière sont représentées dans une majorité d'articles $(n=12)$ et dans une part mineure des mémoires $(n=5)$. Ces résultats vont dans le sens de recherches antérieures qui ont pu montrer que le maniement de cette modalité discursive pouvait en effet être significative des scripteurs aguerris ${ }^{9}$ (voir: BOCH ; GROSSMAN ; RINCK, 2006 ; CORACINI, 1992). On pourrait alors se demander s'il est pertinent de constituer cette catégorie comme relevant d'une référence non théorique lorsqu'il s'agit justement d'une réalisation typique d'un mode de positionnement dans le débat théorique.

En retirant cette catégorie de notre grille d'analyse, la part globale des références non théoriques décrite dans la partie 3.1. passerait de 38,5\% à 34,3\%. Dans ce cas, cette fréquence resterait quasiment inchangée pour les mémoires (de $40 \%$ à $39 \%$ ), mais la variation serait beaucoup plus forte pour les entrées en matière d'article avec une baisse de 10 points (de $35 \%$ environ à $25,6 \%$ ). On obtiendrait dès lors deux taux de référence non théoriques nettement différents. Le rôle dans le positionnement scientifique de cette catégorie de référence à un champ de recherche large sans auteurs cités permet donc de relativiser les convergences décrites plus haut concernant l'importance quantitative dans les deux corpus, des références non théoriques.

${ }^{9}$ C'est peut-être aussi cette position scientifique qui explique le fait que la part des références prescriptives et axiologiques dans le tableau 1 soit plus importante pour les entrées en matière d'articles (respectivement $11,7 \%$ et $6,6 \%$ ) que pour celles des mémoires $(8,7 \%$ et $2,6 \%$ ). Cela pourrait être également en relation avec le fait que les didactiques peuvent se caractériser par des visées qui ne sont pas seulement théoriques mais aussi praxéologiques, sociétales, voire axiologiques. 
Bilan et discussion

Les explorations de nos corpus nous ont permis de rendre compte de plusieurs résultats qui nous semblent intéressants, au premier rang desquels se trouve le fait que les extraits étudiés, qu'ils proviennent d'articles ou de mémoires en didactique, comportent une part relativement importante de ce que nous avons catégorisé comme des références non théoriques. Ces premières observations conduisent donc à penser que ces modalités discursives ne sont, dans les didactiques, ni insolites ni hors-norme et que la littérature scientifique, dans ce domaine en tout cas, n'est pas exempte de références à des points de vue non théoriques et non théorisés.

Nous avons aussi pu montrer que ces références manifestaient des convergences formelles par leur nombre (rapporté à la longueur des textes), leur taille médiane et la variabilité de celle-ci. Ce dernier résultat entre en résonance avec des observations similaires, qui peuvent faire apparaitre une proximité entre les pratiques expertes et néophytes, comme pour les formes de références aux auteurs (BOCH ; GROSSMANN, 2002) ou l'usage de la narration dans un écrit théorique (GEISLER, 1994, cité par DONAHUE, 2007, p. 59). Ces auteurs suggèrent que c'est souvent à un grain plus fin de l'analyse que les différences sont observables.

C'est également ce que nous avons pu montrer, si l'on quitte l'approche strictement quantitative ou que l'on pondère cette dernière en prenant en compte la nature des références. À cet égard, il faut noter une différence importante des types de références selon les corpus : dans les entrées en matière des étudiants, dominent les références à des pratiques d'un champ professionnel ou à des pratiques sociales, à un contexte académique et à un contexte institutionnel; du côté des articles, si les références à un contexte académique ou institutionnel ne sont pas rares, ce sont les références à un champ de recherche large sans auteurs qui étaient les plus représentées.

Nous avons cependant également pu montrer que toutes les catégories de références non théoriques n'étaient pas également représentées dans nos corpus : ainsi, les références à du discours commun, à un intérêt personnel, les références historiques, médiatiques, littéraires et à un lecteur sont apparues comme très rares et même absentes des entrées en matière d'articles pour les trois dernières.

Au-delà de la taille des entrées en matière, la différence entre scripteurs étudiants et scripteurs aguerris semble donc, d'après ces premiers résultats, résider moins dans le fait de faire ou non des références non théoriques, ni dans le nombre global de ces références, ni dans la manière d'y faire référence, mais plutôt dans le type de référence mobilisé. Observons à cet égard que si les références à un champ de recherche large sans auteurs cités, qui identifient la construction scripturale d'une position scientifique affirmée $(\mathrm{BOCH}$; GROSSMANN ; RINCK, 2006), permettent de distinguer les deux corpus, c'est en même temps leur importance en nombre dans les articles qui permettent de faire converger ces écrits 
d'experts et les écrits d'apprentis-chercheurs quant au nombre des références non théoriques.

D'autres recherches devront être entreprises pour tester la portée de ces analyses sur des corpus différents. Mais elles permettent de souligner, si besoin était, toute la nécessité de travaux empiriques pour mieux connaitre les fonctionnements effectifs de l'écriture théorique et dépasser représentations normatives et idéalisées de celle-ci.

\section{Pour un usage didactique de la comparaison}

Si la comparaison binaire peut être euristique d'un point de vue descriptif, elle peut aussi être un outil efficace en contexte didactique; mais, de même que nous pointions les risques de réification d'une comparaison qui figerait des catégories, il ne faut pas mésestimer ses effets pervers bien connus dans une pratique d'enseignement. Le premier est d'encourager subrepticement (sinon explicitement) l'institution d'une norme implicite de l'écrit à produire, qui présente au moins deux faiblesses : d'une part, elle suppose une homogénéité du discours scientifique qui n'est pas avérée, comme on l'a vu; d'autre part, et surtout, elle néglige que le discours d'un débutant a précisément ses normes propres, qui ne sont pas celles du discours de l'expert (cf. DONAHUE, 2008, p. 211, qui montre le caractère propre du " discours d'apprentissage $»)$.

Le deuxième effet pervers possible est de construire des modèles inatteignables, qui supposent une rupture entre l'écrit de l'étudiant et celui de l'expert, au lieu de l'envisager dans les termes plus complexes du passage (DELAMOTTE et al., 2000) que permet de concevoir la notion de continuum avancée par DABÈNE (1987) : il devient alors possible d'envisager des variations de contextes qui peuvent mettre tout scripteur dans une plus ou moins grande situation d'inexpertise.

Nous abordons ici trois activités menées dans nos cours à partir du corpus analysé cidessus. Nous ne présentons pas ces activités comme des résultats de recherche, au sens où nous ne les avons ni construites à partir d'un état de l'art spécifique ni étudiées en suivant une méthodologie rigoureuse : il faut les prendre ici comme des suggestions qui nous sont venues à l'esprit à partir de notre travail d'analyse du corpus, autrement dit, pour jouer sur les mots, comme un résultat de notre recherche - à mettre en discussion avec d'autres propositions de travail issues de la recherche sur l'écriture scientifique en formation (cf. BOCH ; FRIER, 2015 ; POLLET, 2014). 


\section{Références canoniques?}

La question du titre est évidemment ironique, dans la mesure où, dans le domaine qui nous concerne, le canon d'une écriture scientifique n'est pas si facile à déterminer... Mais elle dit en même temps que nous avons bien affaire à une question de norme : c'est l'enjeu d'un travail avec des étudiants de les amener à penser cette question, même si une norme se laisse difficilement décrire de manière univoque (BART, 2013). De fait, les extraits qui suivent, supports de notre exercice, s'approchent ou s'éloignent, selon les cas, de ce qui nous semble être la norme dominante en vigueur en matière de référence. On pourrait en discuter : et c'est précisément ce qu'il est question de faire dans cette activité...

L'exercice a un triple objectif : identifier différents types de références dans des entrées en matière (les plus fréquents selon les observations dont rendent compte les tableaux cidessus) ; interroger leur légitimité ; observer la proximité possible entre une écriture experte et une écriture débutante. C'est pour cela, du reste, que nous avons volontairement sélectionné des extraits d'articles qui peuvent susciter la discussion et des extraits de mémoires qui ne semblent pas poser de problème ; cela obéit à un principe que nous mettons en œuvre dans nos cours : si nous donnons des travaux d'étudiants à lire, c'est en général pour identifier ce qui fonctionne et s'il est utile de recourir à des contrexemples, nous les empruntons plutôt à des travaux publiés (y compris les nôtres)...

Le choix des extraits a été effectué en fonction des intentions didactiques (exemples représentatifs et contrastés, notamment) mais aussi pour des raisons pratiques (clarté et longueur, par exemple); nous avons par ailleurs évité les extraits qui identifiaient explicitement leur origine (« cet article », « ce mémoire »...).

Voici le matériau donné aux étudiants (les extraits sont classés par ordre alphabétique).

$\mathrm{n}^{\circ} 1$ Ce travail est la poursuite de nos questionnements autour de la construction de savoirs scientifiques par les élèves, en gardant comme préoccupation les persistances de conceptions erronées témoignant des difficultés qui peuvent exister.

$\mathrm{n}^{\circ} 2$ Ces dernières années ont vu s'installer durablement dans le paysage éducatif ce que I'on a d'abord appelé dans la langue de l'institution scolaire les « nouvelles technologies de l'information et de la communication » (NTIC), les « technologies de l'information et de la communication " (TIC) puis plus récemment les « technologies usuelles de l'information et de la communication » (TIUC).

$\mathrm{n}^{\circ} 3$ Il est donc tout à fait intéressant d'interroger cette rencontre entre l'École et le Musée.

$\mathrm{n}^{\circ} 4$ La lecture des analyses sur les évaluations nationales faites par le Ministère de l'éducation nationale depuis 1989 montre que les élèves peuvent avoir, pour 80\% d'entre eux, une bonne compréhension des consignes simples. C'est un pourcentage honorable mais rappelons qu'il s'agit d'élèves de cycle 3 et que cette compétence est travaillée depuis la section des petits de l'école maternelle. 
$\mathrm{n}^{\circ} 5$ Les liens entre les sciences qui se font et les sciences à l'école constituent une question régulièrement revisitée, notamment à chaque changement de paradigme concernant les sciences ou leur enseignement-apprentissage.

$n^{\circ} 6$ Néanmoins, nous tenons à préciser que nous ne nous situons pas dans l'axe de recherche des pratiques enseignantes qui a pour objectif de mieux appréhender l'efficacité de l'enseignement sur les apprentissages.

$\mathrm{n}^{\circ} 7 \mathrm{Ni}$ motif de sanction telle que le redoublement, ni critère de sélection - ce qu'illustrent les très faibles coefficients et volumes horaires affectés à l'éducation musicale -, cette discipline se situe en périphérie des enjeux scolaires. On peut donc penser que cette structure d'incitation favorise l'émergence de tensions entre le rapport à la musique défendu par l'institution scolaire et les pratiques d'écoute ordinaires des élèves.

$n^{\circ} 8$ Nous avons recueilli des données en 2008, au moment où la mastérisation allait se mettre en place, auprès de Professeurs des Écoles stagiaires (PES) qui avaient, comme les PES actuels, des périodes de Pratique accompagnée (PA) dans les classes des Professeurs des Écoles Maitres-Formateurs (PEMF).

$\mathrm{n}^{\circ} 9$ Répondre à cette double exigence invite donc à concevoir la lecture des textes littéraires dès l'école maternelle comme le tissage d'un rapport intime à la lecture et comme une fabrique à souvenirs de lecture.

$\mathrm{n}^{\circ} 10 \quad \mathrm{~S}^{\prime} \mathrm{il}$ permet au jeune lecteur à la fois d'exprimer l'unicité de son questionnement et de développer un rapport plus authentique à la lecture, le journal dialogué nous apparait aussi comme un puissant outil d'enseignement, en ce qu'il permet à l'enseignant de lier appétence et compétence et surtout de lire les divers " évènements " et obstacles de lecture survenant dans le contexte réel de sa classe.

$n^{\circ} 11$ Une description de différente pratiques enseignants de l'évaluation peut permettre d'éclairer le champ des possibles, à la fois pour les enseignants et les formateurs et d'aider à la réflexion sur le fonctionnement du système évaluatif et les difficultés des élèves en situation d'évaluation.

$n^{\circ} 12$ Une équation du premier degré en $x, y$ représente une droite dans un plan muni d'un repère $0 x y$ et, dans l'espace usuel muni d'un repère $O x y z$, un plan parallèle à l'axe $O z$.

Voici, dans le tableau qui suit, l'origine de ces extraits. Nous les donnons ici d'une part pour permettre d'identifier à quels corpus (articles ou mémoires) appartiennent les extraits et parce que cela pourra permettre d'exemplifier quelques-unes de nos catégories (il faut bien sûr garder en mémoire les contraintes et les critères qui ont présidé à nos indexations). Mais ce tableau n'a pas vocation à être fourni aux étudiants. 
Tableau 3. Répartition des extraits de l'exercice 1 (entre parenthèses : code de la source dans le corpus)

\begin{tabular}{|c|c|c|}
\hline Références & Articles & Mémoires \\
\hline Pratiques & $\mathrm{n}^{\circ} 7(\mathrm{~A} 15)$ & $\mathrm{n}^{\circ} 2(\mathrm{M} 7)$ \\
\hline Contexte académique & $n^{\circ} 8(A 2)$ & $\mathrm{n}^{\circ} 1(\mathrm{M} 12)$ \\
\hline Contexte institutionnel & $\mathrm{n}^{\circ} 5(\mathrm{~A} 6)$ & $\mathrm{n}^{\circ} 4(\mathrm{M} 6)$ \\
\hline Champ de recherche large & $\mathrm{n}^{\circ} 12$ (A12) & $\mathrm{n}^{\circ} 6$ (M5) \\
\hline Futur & $n^{\circ} 9$ (A9) & $\mathrm{n}^{\circ} 11(\mathrm{M} 11)$ \\
\hline Valeurs & $\mathrm{n}^{\circ} 10(\mathrm{~A} 7)$ & $\mathrm{n}^{\circ} 3(\mathrm{M} 20)$ \\
\hline
\end{tabular}

La première consigne est de demander aux étudiants quels sont les extraits qui leur semblent acceptables dans l'entrée en matière d'un discours scientifique. Ce travail (individuel ou en groupe, ou les deux successivement) n'a pas, répétons-le, pour fonction de donner une règle définitive (sachant que les articles publiés dans des revues reconnues montrent une réelle diversité de pratiques), mais simplement - et au moins - d'amener à identifier que des choix discursifs qui pourraient leur sembler naturels peuvent pourtant être discutables. Les réponses des étudiants (dans le travail initial ou dans la discussion collective qui s'ensuit) ainsi que le guidage de l'enseignant dépendront notamment du moment de l'exercice, selon que les étudiants auront ou non déjà identifié le statut plus ou moins légitime de tels ou tels types de discours dans un écrit scientifique.

Suite à cette activité, les étudiants sont invités (dans les mêmes modalités de travail) à nommer les types de références en jeu. L'objectif n'est pas de retrouver nos catégories, mais de catégoriser... Du reste, la chose n'est pas simple puisque de nombreux extraits peuvent entrer dans diverses catégories. L'objectif est ici de construire avec les étudiants la notion de référence du discours à des champs d'expérience autres que les discours d'auteurs théoriques.

Enfin, la dernière consigne est d'identifier les extraits d'articles et de mémoires : là encore, la réponse n'a en elle-même aucune importance, mais cette discussion est une autre manière d'interroger les normes, tout en faisant ressortir les possibles proximités entre une écriture experte et une écriture débutante. 


\section{Références privilégiées}

Sans décrire dans le détail les deux autres activités, nous en donnerons succinctement les principes et suggèrerons les objets possibles de discussion avec les étudiants. La première peut avoir lieu, si l'on pense en termes de progression didactique, avant celle que nous venons de décrire, comme elle peut la suivre. Elle n'est pas directement issue des résultats de notre étude, fondée sur la quantification des items sélectionnés, mais se veut précisément complémentaire, en ce qu' elle demande aux étudiants une analyse plus qualitative fondée sur une approche textuelle : la comparaison de deux entrées en matière issues respectivement de nos deux corpus (nous ne reproduisons pas d'exemples ici, faute de place mais aussi d'intérêt : c'est plutôt dans l'environnement théorique et institutionnel des étudiants que le choix de ces deux extraits peut prendre sens). L'objectif est double : d'une part, amorcer ou redoubler (selon la place de l'activité par rapport à celle décrite ci-dessus) le travail de catégorisation des références en jeu (les extraits choisis devant en comporter plusieurs) ; d'autre part, faire apparaitre une différence entre les entrées en matière, certaines pouvant être considérées comme assez éloignées de l'écriture attendue, moins par le fait de s'appuyer sur des références non théoriques que par l'absence des moyens rhétoriques permettant de les enchâsser dans un discours théorique et de les mettre en relation avec des préoccupations d'une communauté de recherche.

Une telle analyse permet de compenser les défauts d'une réflexion à partir d'extraits décontextualisés, qui peuvent réifier le contenu en jeu et, contre la logique même des objectifs poursuivis, laisser supposer que l'acculturation à un discours scientifique peut se faire par le respect de normes strictement techniques et que l'acquisition d'une compétence globale pourrait se satisfaire de l'accumulation de compétences locales...

La deuxième activité (dont nous pensons qu'elle a mieux sa place après les activités décrites précédemment) consiste à faire analyser par les étudiants le tableau 2 , avec le double objectif de faire ressortir les divergences entre les articles et les mémoires, non pour stigmatiser ceux$\mathrm{ci}$, mais pour interroger les raisons possibles de ces divergences.

Dans un premier temps, une rapide description de notre recherche est faite aux étudiants, ainsi que la présentation des premiers résultats (que faisait apparaitre le tableau 1) : si les entrées en matière d'articles et de mémoires se distinguent par leur longueur, elles convergent par certains aspects formels; une conclusion possible de cette observation est qu'elles ont leur place dans la littérature scientifique didactique et que la présence de cellesci ne disqualifie pas à priori un écrit.

L'analyse du tableau 2 avec les étudiants permet de pondérer cette observation et de mieux faire ressortir des divergences, intéressantes dans une perspective d'apprentissage des normes de l'écriture de recherche. Les consignes de ce travail (qui peut être fait seul, en groupe ou dans un échange collectif) sont simples et assez vagues pour ne pas enfermer la 
discussion : « Identifiez au moins deux convergences et deux différences entre les deux corpus et proposez-en des interprétations possibles ". Deux aspects, présentés ci-dessus, peuvent ressortir de l'analyse - outre l'imprévisible qui peut naitre des interactions en cours, évidemment ! D'une part, les types de références, qui différencient les deux corpus ; d'autre part, l'absence de certains types de références dans les articles, qu'on voit représentées dans les mémoires.

Ces divergences peuvent trouver quelques explications, que notre recherche ne peut confirmer, mais qui peuvent faire l'objet d'une discussion avec les étudiants : l'usage de ces références non théoriques par les étudiants serait-il le signe d'un apprentissage de l'écriture de recherche et du passage d'une forme d'inscription pratique, académique ou institutionnelle de la recherche à une forme d'inscription distanciée dans les cadres du dialogue théorique? Ces formes d'inscription non théoriques constitueraient alors des ressorts scripturaux offrant l'élan nécessaire à l'entrée dans l'écrit de recherche lorsque les soubassement d'un positionnement théorique ne sont pas encore construits. Et sans doute ne faut-il pas négliger l'intérêt de l'explicitation des relations personnelles ou pratiques à une thématique qui peut faire l'objet d'un questionnement de recherche : ce pourrait en effet être un moyen de clarifier les présupposés, convictions, croyances, représentations, expériences du scripteur et de les prendre pour objet pour mieux les mettre à distance dans l'écriture de recherche.

\section{Conclusion}

Notre travail ici même n'avait pas pour intention d'épuiser la question et nous nous sommes contentés de l'indication de quelques implications pratiques d'une étude fondée sur une approche quantitative. Ces activités que nous avons présentées ou évoquées illustrent toutes un principe bien décrit par Boch (2013, p. 544) :

Notre hypothèse de travail repose sur l'idée qu'une formation à l'écriture scientifique doit prioritairement passer par la conscientisation des pratiques en usage (plutôt que par un discours prescriptif, insuffisant pour modifier en profondeur les comportements), en permettant aux jeunes chercheurs (et aux formateurs qui les encadrent) de s'approprier les caractéristiques énonciatives du genre, qui demeurent souvent intuitives ou peu formalisées.

Nous ajouterons que ces pratiques en usage sont autant celles d'experts que de néophytes.

La comparaison entre les écrits de débutants et les écrits de ceux qui ont un jour, comme eux, débuté dans l'écriture de recherche, outre les effets de compréhension qu'elle peut engendrer, permet de mettre au jour un continuum qui les inscrit, dès l'abord, dans la 
communauté des chercheurs entendue comme une "communauté de pratiques " (LAVE ; WENGER, 1991).

\section{Références}

BAILLY, S.; ALVES ASSIS, J. ; DENEI, M. Écrire et apprendre à écrire à l'université brésilienne, française et québécoise : questions de recherche en didactique des langues, Mélanges CRAPEL, Nancy, v. 37, n. 1, p. 5-9, 2016.

BART, D. La direction des thèses de "futurs collègues " en sciences de l'éducation : un accompagnement doctoral entre formation au travail autonome et intégration à un collectif scientifique. In: FRENAY M. ; ROMAINVILLE M. (Dir.). L'accompagnement des mémoires et des thèses. Louvain: Presses Universitaires de Louvain, 2013, p. 109-128.

$\mathrm{BOCH}, \mathrm{F}$. Former les doctorants à l'écriture de la thèse en exploitant les études descriptives de l'écrit scientifique. Linguagem em (Dis)curso, Santa Catarina, v. 13, n. 3, p. 543-568, 2013. https://doi.org/10.1590/S1518-76322013000300005

BOCH, F.; FRIER, C. (Dir.). Écrire dans l'enseignement supérieur : des apports de la recherche aux outils pédagogiques. Grenoble: UGA Éditions, 2015. https://doi.org/10.4000/books.ugaeditions.1438

BOCH, F.; GROSSMANN, F. Se référer au discours d'autrui : comparaison entre experts et néophytes. Enjeux, Namur, n. 54, p. 41-51, 2002.

BOCH, F.; GROSSMANN, F.; RINCK, F. Le cadrage théorique dans l'article scientifique : un lieu propice à la circulation des discours. In: LÔPEZ MUNOZ, J.M.; MARNETTE S.; ROSIER L. (Dir.). Colloque Ci-Dit Circulation des discours et liens sociaux : le discours rapporté comme pratique sociale. Québec: Nota Bene, 2010, p. 23-42.

BOCH, F.; RINCK, F. Pour une approche énonciative de l'écrit scientifique. Lidil, Grenoble, n. 41, p. 5-14, 2010.

BOLÍVAR, A.; PARODI, G. Academic and Professional Discourse. In: LACORTE M. (Dir.). The Routledge Handbook of Hispanic Applied Linguistics. New York and London: Routledge, Taylos and Francis, 2015, p. 459-476.

CORACINI, M. J. R. F. L'hétérogénéité dans un discours scientifique français et brésilien : un effet persuasif. Langages, Paris, n. 105, p. 76-86, 1992. https://doi.org/10.3406/lgge.1992.1625

CRINON, J. et GUIGUE, M. Être sujet de son écriture : une analyse de mémoires professionnels. Spirale, Lille, n. 29, p. 201-219, 2002. https://doi.org/10.3406/spira.2002.1445

DABÈNE, M. L'adulte et l'écriture: contribution à une didactique de l'écrit en langue maternelle. Bruxelles: De Boeck-Wesmael, 1987.

DAUNAY, B.; DELCAMBRE, I. Les modalités énonciatives de la reprise du discours d'autrui dans les écrits de recherche et les écrits didactiques. Scripta, Belo Horizonte, v. 21, n. 43, p. 37-64, 2017. https://doi.org/10.5752/P.2358-3428.2017v21n43p37 
DELAMOTTE, R. et al. Passages à l'écriture. Paris: PUF, 2000.

DELCAMBRE I. Pour une didactique des disciplines universitaires ? In: POLLET M.-C.; GLORIEUX C. (Dir.). Argumenter dans les écrits scientifiques. Namur: Presses universitaires de Namur, 2016, p. 213-222.

DELCAMBRE, I. et LABORDE-MILAA, I. Diversité des modes d'investissement du scripteur dans l'introduction du mémoire professionnel. Enjeux, Namur, n. 53, p. 11-22, 2002.

DELCAMBRE, I.; LAHANIER-REUTER, D. Les littéracies universitaires : Influence des disciplines et du niveau d'étude dans les pratiques de l'écrit. www.forumlecture.ch, n. 3, 2010. Disponible à $\quad$ https://www.forumlecture.ch/sysModules/obxLeseforum/Artikel/431/Les-litteraciesuniversitaires.pdf>. Consulté le 12 fév. 2019.

DETIENNE, M. Comparer l'incomparable. Paris: Seuil, 2000.

DONAHUE, C. Écrire à l'université : analyse comparée en France et aux Etats-Unis. Villeneuve d'Ascq: Presses universitaires du Septentrion, 2008. https://doi.org/10.4000/books.septentrion.14852

DONAHUE, C. Évolution des pratiques et des discours sur l'écrit à l'université : étude de cas. Lidil, Grenoble, n. 41, p. 137-160, 2010. https://doi.org/10.4000/lidil.3034

FL $\varnothing T T U M, K$. Moi et autrui dans le discours scientifique : l'exemple de la négation « ne... pas ». In BRES J. (Dir.). Dialogisme et polyphonie: approches linguistiques. Louvain-la-Neuve: De Boeck, 2005, p. 323-337.

FLØTTUM K.; DAHL, T.; KINN, T. Academic Voices. Across languages and disciplines. Amsterdam/Philadelphia: John Benjamins, 2006. https://doi.org/10.1075/pbns.148

FLØTTUM, K.; VOLD, E. T. L'éthos auto-attribué d'auteurs-doctorants dans le discours scientifique. Lidil, Grenoble n. 41, p. 41-58, 2010. https://doi.org/10.4000/lidil.3006

FRANÇOIS, F. Bakhtine tout nu. Limoges: Lambert-Lucas, 2012.

GEISLER, C. Academic literacy and the nature of expertise: reading, writing, and knowing in academic philosophy. Hillsdale N.J.: Lawrence Erlbaum Associates, 1994.

GROSSMANN, F. Objectivité scientifique et positionnement d'auteur. In: Schnedecker C.; ALEKSANDROVA A. (Dir.). Le doctorat en France : mode(s) d'emploi. Bruxelles: Peter Lang, 2017a, p. 97-112.

GROSSMANN, F. Vingt ans de travaux sur l'écriture de recherche. Quel bilan pour préparer l'avenir ? In: Dias-Chiaruttini A.; Cohen-Azria C. (Dir.). Théories-didactiques de la lecture et de l'écriture : fondements d'un champ de recherche, en cheminant avec Yves Reuter. Villeneuve d'Ascq: Presses universitaires du Septentrion, 2017b, p.111-134. https://doi.org/10.4000/books.septentrion.15251

GROSSMANN, F.; RINCK, F. La surénonciation comme norme du genre : l'exemple de l'article de recherche et du dictionnaire en linguistique. Langages, Paris, v. 38, n. 156, p. 34-50, 2004. https://doi.org/10.3406/lgge.2004.962

HERMAN, T. De la captatio à la partitio: rhétorique de l'introduction de l'article de recherche. Travaux neuchâtelois de linguistique, Neuchâtel, n. 65, p. 89-111, 2017. 
HYLAND, K.; BONDI M. (Dir.). Academic discourse across disciplines. New York: Peter Lang, 2006. https://doi.org/10.3726/978-3-0351-0446-2

JACQUES, M.-P. La structuration textuelle en discours scientifique : comparaison oral / écrit. CHIMERA. Romance Corpora and Linguistic Studies, Madrid, n. 1, p. 89-115, 2017.

KANTÉ, I. Head nouns as modal stance markers. Academic texts vs. legal texts. Lidil, Grenoble, n. 41, p. 121-135, 2010. https://doi.org/10.4000/lidil.3022

KLEIBER G. Sens, référence et existence : que faire de l'extra-linguistique ?. Langages, n. 127, p. 9-37, 1997. https://doi.org/10.3406/lgge.1997.2123

LAVE J.; WENGER E. Situated learning: Legitimate Peripheral Participation. Cambridge: Cambridge University Press; 1991. https://doi.org/10.1017/CB09780511815355

MOIRAND S. L'évaluation dans les discours scientifiques et professionnels. Les Carnets du Cediscor, Paris, n. 3, p. 81-93, 1995.

PASQUOTTE-VIEIRA, E. A.; SALEK FIAD, R. Letramentos acadêmicos: entre práticas letradas acadêmicas e não acadêmicas. Linguagem \& Ensino, Pelotas, v.18, n.1, p. 125-150, 2015.

POLLET, M.-C. L'écrit scientifique à l'aune des littéracies universitaires : approches théoriques et pratiques. Namur : Presses universitaires de Namur, 2014.

POLLET, M.-C.; GLORIEUX, C. (Dir.). Argumenter dans les écrits scientifiques. Namur: Presses universitaires de Namur, 2016.

REUTER, Y. Je suis comme un autrui qui doute. Le discours des autres dans l'écrit de recherche en formation. Lidil, n. 25, p. 13-28, 2001.

REUTER, Y. Analyser les problèmes de l'écriture de recherche en formation. Pratiques, Metz, n. 121-122, p. 9-27, 2004. https://doi.org/10.3406/prati.2004.2029

REUTNER, U.; SCHWARZE, S. (Dir.). Le style, c'est l'homme: unité et pluralité des discours scientifique. Francfort-sur-le-Main, Peter Lang, 2008.

RINCK, F. L'article de recherche en Sciences du Langage et en Lettres : figure de l'auteur et approche disciplinaire du genre. Thèse de doctorat, Université de Grenoble, 2006.

RINCK, F.; POUVREAU, L. La mise en scène de soi dans un écrit d'initiation à la recherche en didactique du français. Scripta, Belo Horionte, v. 13, n. 24, p. 157-172, 2009.

RUSSELL, D. R. Écrits universitaires / écrits professionnalisants / écrits professionnels : est-ce qu' « écrire pour apprendre » est plus qu'un slogan ? Pratiques, Metz, n. 153-154, p. 21-34, 2012. https://doi.org/10.4000/pratiques.1913

SWALES, J. Genre Analysis : English in Academic and Research Settings. Cambridge: Cambridge University Press, 1990.

TUTIN, A. "Dans cet article, nous souhaitons montrer que... ". Lexique verbal et positionnement de l'auteur dans les articles en sciences humaines. Lidil, Grenoble, n. 41, p. $15-$ 40, 2010. https://doi.org/10.4000/lidil.3040

TUTIN, A.; GROSSMANN, F. (Dir.). L'écrit scientifique : du lexique au discours. Rennes : Presses universitaires de Rennes, 2013. 
VOLD, E. T. Modalité épistémique et discours scientifique: une étude contrastive des modalisateurs épistémiques dans des articles de recherche français, norvégiens et anglais, en linguistique et médecine, Thèse de doctorat, Université de Bergen, 2008.

Recebido em: 15/02/2019

Aceito em: 04/06/2019 